\title{
Review
}

\section{Older persons' experiences and perspectives of receiving social care: a systematic review of the qualitative literature}

\author{
José de São José $\mathrm{PhD}^{1}$, Rosanna Barros $\mathrm{PhD}^{2}$, Sanda Samitca $\mathrm{PhD}^{3}$ and Ana Teixeira MD ${ }^{1}$ \\ ${ }^{1}$ Faculty of Economics, University of Algarve, Research Centre for Spatial and Organizational Dynamics, Faro, Portugal, \\ ${ }^{2}$ School of Education and Communication, University of Algarve, Faro, Portugal and ${ }^{3}$ Institute of Social Sciences, Uni- \\ versity of Lisbon, Lisbon, Portugal
}

Accepted for publication 27 October 2014

\author{
Correspondence \\ José de São José \\ Faculty of Economics \\ University of Algarve \\ Campus de Gambelas, Edifício 9, \\ 8005-139 Faro, Portugal \\ E-mail: jsjose@ualg.pt
}

\section{What is known about this topic}

- The full understanding of a care relationship requires looking not only to the provider's side but also to the receiver's side.

- The receivers of care, including older people, are not passive actors.

- The literature on the older persons experiences of receiving social care is still limited.

\section{What this paper adds}

- Positive experiences of receiving social care include reciprocity, respect and attentiveness, safety and security, and engagement in decisions concerning care.

- Negative experiences of receiving social care include difficulties in asking for care, ambivalences, disengagement from decisions concerning care and multiple losses.

- Reviewed evidence supports the ethics of care and dignity conceptual frameworks, and demonstrates that care practices and public policies should pay particular attention to the relational dimension of care.

\begin{abstract}
The topic of social care for older people has gained increasing attention from the part of academics, professionals, policy makers and media. However, we know little about this topic from the perspectives of older persons, which hinders future developments in terms of theory, empirical research, professional practice and social policy. This article presents and discusses a systematic review of relevant qualitative research-based evidence on the older persons' experiences and perspectives of receiving social care published between 1990 and September 2014. This review aimed to obtain answers to the following questions: How is the reception of social care experienced by the older persons? What are the negative and positive aspects of these experiences? What are the factors which influence the experiences? The synthesis of the findings of reviewed papers identified six analytical themes: asking for care as a major challenge; ambivalences; (dis)engagement in decisions concerning care; multiple losses as outcomes of receiving social care; multiple strategies to deal with losses originated by the ageing process; and properties of 'good care'. These themes are discussed from the point of view of their implications for theory, care practice and social policy, and future research.
\end{abstract}

Keywords: experiences and perspectives, older people, qualitative studies, receiving care, social care, systematic review

\section{Introduction}

This article presents and discusses a systematic review of qualitative research-based evidence, published between 1990 and September 2014, that examines the experiences and perspectives of older persons receiving social care in the community. We have defined the category 'older persons' as those who are aged 65 and over, given that western societies tend to associate 65 with the beginning of old age (Gorman 2000). Further, 'social care' includes actions directed towards meeting basic needs of daily living, such as feeding and bathing, and also social-emotional needs such as company and moral support (healthcare is excluded). Social care can be provided by people belonging to the formal sector (public, commercial or voluntary sector) or by people belonging to the informal sector (family members, neighbours or friends) (Sipilä \& Kröger 2005).

Currently, the social policy agenda on health and social care for older people is dominated by discourses advocating quality in services, 
ageing-in-place, person-centred care and dignity in later life (e.g. the Health and Social Care Act 2008 in the UK, the European Charter of the Rights and Responsibilities of Older People in Need of Longterm Care and Assistance). These discourses emphasise care receivers' self-determination, choice, independence, involvement and control, although some authors argue that other values beyond independence and choice, such as societal responsibility and interdependence, should be taken into account if we want effective improvements in the quality of life of older people who receive care (e.g. Nolan et al. 2004).

On the other hand, there are theoretical arguments in favour of understanding the care receivers' experiences and perspectives. For example, in 1993, Joan Tronto proposed a theoretical/philosophical perspective on care, later followed by others (e.g. Sevenhuijsen 1998, Engster 2007), which recognises that a full understanding of the caring process requires an analytical focus not only on the carers' side but also on the care receivers' side. This understanding is based on the principle that receivers of care, including older people, are not passive actors, as demonstrated by some empirical research (e.g. Twigg 2000, Lloyd et al. 2014).

Developing better care for older people is the main promise of the policy discourses mentioned earlier, but it will be very difficult to develop better care without a full understanding of the elders' experiences and perspectives of receiving care. There is some empirical research and policy analysis that focuses on capturing these experiences and perspectives, but this is still scanty (Edmondson \& Kondratowitz 2009, Lloyd 2012).

Thus, we claim that to inform policy and practice (professional and non-professional) with the ultimate goal of improving care, and also to guide future developments in theory and empirical research in this particular field, we need to have a comprehensive synthesis of the elders' experiences and perspectives of receiving social care. In this sense, we opted for undertaking a systematic review of the literature (only qualitative research) and not a narrative review on the topic of social care for older people, given that the aim is not summarising 'all there is to know' (Petticrew \& Roberts 2006, p. 10) about this topic, but rather to get answers to a set of succinct review questions (presented in the next section).

Before we move on to the next section, it is important to say something about two conceptual frameworks focused on the topic of care for which this review may be particularly relevant. The first one is the ethics of care perspective initially proposed by Tronto (1993) and the second one is the model of dignity proposed by Nordenfelt (2009). Tronto (1993) advocates that the 'integrity of care' can be achieved only through the integration of four moral principles: attentiveness, responsibility, competence and responsiveness. Sevenhuijsen (1998) proposes trust as a fifth principle, and Engster (2007) proposes respect as yet another principle. In addition, Nordenfelt (2009) proposes four forms of dignity - human dignity, dignity of merit, dignity of moral stature and dignity of identity - and considers the last form to be most likely the most important in the context of ageing and care. Nordenfelt 2009, p. 33 defines dignity of identity as:

(...) the dignity that we attach to ourselves as integrated and autonomous persons, with a history and a future, with all our relationships to other human beings.

However, these two conceptual frameworks are scarcely used in current empirical research, so they lack consistent empirical support (Gallagher et al. 2008, Barnes 2012). Therefore, this systematic review may contribute to clarify if the existing empirical research on the older persons' experiences of receiving social care supports these conceptual frameworks or if the research supports other perspectives.

\section{Overall aim and review questions}

The overall aim of this systematic review was to identify, appraise, synthesise and discuss relevant research-based evidence of the experiences and perspectives of older persons receiving social care in the community. We intend to obtain answers to the following questions:

- How do older persons experience the reception of social care?

- What are the negative and positive aspects of these experiences?

- What are the factors that influence the experiences?

\section{Methods}

The best methods for undertaking a systematic review of qualitative empirical research are still under debate (Britten et al. 2002, Thomas \& Harden 2008). We adopted the 'SCIE Systematic Research Reviews: guidelines' (SCIE 2010) as a general framework to identify and appraise the studies.

\section{Inclusion and exclusion criteria}

On the basis of the overall aim of this systematic review and associated questions, we used the following 
basic criteria for inclusion of empirical studies (a full identification, description and justification of the inclusion and exclusion criteria can be found in Table S1 of the supporting material in the online version of this article):

- a focus on older people (people aged 65 and over);

- a focus on older people living in the community (not in institutional settings);

- a focus on older people who were receiving social care at the moment of data collection (or who had stopped receiving it less than 1 year previously);

- an account of the experiences and perspectives of these older people;

- a base on qualitative research (data collection and analysis);

- a focus on older people living in one of the countries of the European Union (27 countries).

Only studies of older people living in the community were included in the literature review because we surmised that the inclusion of studies of older people living in institutional settings would create some difficulties in the review process. For example, more heterogeneity would be added to the existing heterogeneity found in the studies that include only non-institutionalised older persons; furthermore, the volume of studies to screen would be considerably increased.

\section{Search strategies}

We employed several search strategies to identify potential, relevant studies, including the following: searching of electronic databases, hand searching of electronic journals, reviewers' knowledge of the literature and citation tracking. We searched the following electronic databases: Social Science Citation Index (SSCI, CPCI-SSH, BKCI-SSH), PsycINFO, MEDLINE, System for Information on Grey Literature in Europe (SIGLE), Google Scholar and Porbase. Several combinations of keywords in English were used to search in all databases: old* people/elder* people and receiv* care/care*relation*/provider relation* and experien*/opinion*/view*/perception*/attitude*/satisf*/meaning*.

Hand searching of electronic journals was conducted to increase the probability of including the latest published studies. We hand searched the last issues of three prestigious, European-based journals on Gerontology and Social Care (content pages: title and abstract): Ageing and Society, Health and Social Care in the Community and the Scandinavian Journal of Caring Sciences.

\section{Paper selection process}

Our initial searches covered papers published between 1990 and 2012. At a later stage, we updated these searches focusing on papers published between 2013 and September 2014. The initial searches identified 29 papers meeting the inclusion criteria, while the last searches identified one paper meeting the inclusion criteria. In total, 30 papers (of 24 studies) published between 1990 and September 2014 were included in this review (see Figure 1).

\section{Quality rating}

Based on the 'SCIE Systematic Research Reviews: guidelines' (SCIE 2010), different parameters were taken into account for extracting data, including quality rating (appraisal of the quality of the studies). The majority of the reviewed papers obtained a high quality rating, and the remaining ones obtained a medium quality rating but were close to the highest rating. The overall quality rating was calculated by attributing a score to five parameters: aims, sampling, methods, analysis and relevance to the review questions (see Box 1).

\section{Synthesis of papers' findings}

We synthesised the papers' findings by using the approach designated by 'thematic synthesis', as proposed and described in detail by Thomas and Harden (2008). The 'thematic synthesis' is a tested technique based on three stages: line-by-line coding, organisation of 'descriptive' themes and generation of 'analytical' themes. In the first stage, the aim is to code each line of the text reporting the information about each reviewed paper (in our case, the coded text corresponds to the information inserted in the grid of extracting data). This initial coding produces 'free codes', which are grouped into 'descriptive' themes in the second stage. For example, all the 'free codes' which denoted practices of giving and receiving in care relationships were grouped into a descriptive theme designated as 'reciprocal interactions'. In the third stage, the aim is 'going beyond' the findings of reviewed papers through the generation of 'additional concepts, understandings or hypotheses' (Thomas \& Harden 2008, p. 7). This aim is achieved using the descriptive themes to answer the review questions. For example, the descriptive theme 'reciprocal interactions' was integrated into the analytical subtheme 'reciprocity' which, in turn, is an integral part of the analytical theme 'properties of good care'. The synthesis was supported by the software NVivo 9 . 


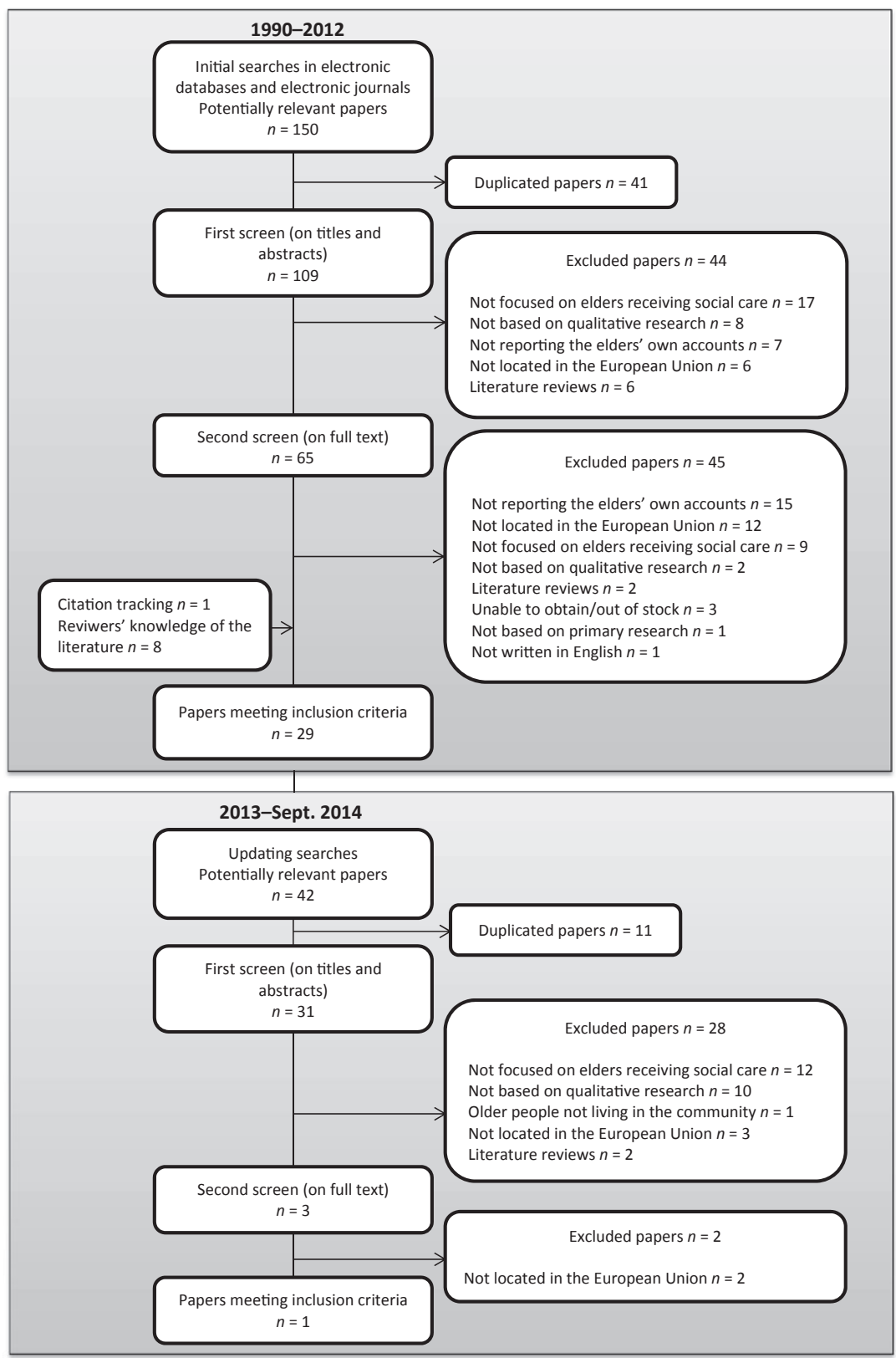

Figure 1 Flow chart of paper selection process.

\section{General overview of the included studies}

Before presenting the analytical themes and subthemes, it is important to make some distinctions about the reviewed papers. Most of the papers are based on studies conducted in the United Kingdom, and the remaining ones are based on studies conducted in Scandinavian countries, predominantly Sweden. Only one study used samples collected in more than one country (Tadd \& Calnan 2009). The reviewed studies are relatively recent; the majority were published after 2005, and only one was published before 2000.
Moreover, the studies used a great variety of theoretical and methodological approaches (although some were not explicitly identified). Concerning sample composition, with the exception of a few cases, the reviewed studies were heterogeneous in terms of age, gender and marital status. With the exception of one case (Forbat 2005), all samples included older persons receiving some kind of formal care in the community (usually home care), with most of them combining this kind of care with informal care (usually family care). It is important to add that a few of the samples included some older persons living in sheltered hous- 
Box 1 Appraisal of the papers' quality

\author{
Parameter A: Aims \\ 1. Research aims are: \\ a. Explicitly stated \\ b. Implicitly stated \\ c. Not stated \\ d. Unclear \\ Parameter B: Sampling \\ 2. Sampling strategies and procedures are: \\ a. Explicitly stated \\ b. Implicitly stated \\ c. Not stated \\ d. Unclear \\ 3. Sample composition (characteristics of participants) is: \\ a. Explicitly stated \\ b. Implicitly stated \\ c. Not stated \\ d. Unclear \\ Parameter C: Methods \\ 4. Data collection tools are: \\ a. Explicitly stated \\ b. Implicitly stated \\ c. Not stated \\ d. Unclear \\ 5. Data analysis tools are: \\ a. Explicitly stated \\ b. Implicitly stated \\ c. Not stated \\ d. Unclear \\ Parameter D: Analysis \\ 6. Does the paper discuss the quality of the analysis/ \\ findings? \\ a. Yes \\ b. Partially \\ c. No \\ d. Unclear \\ Parameter E: Relevance to the review questions \\ 7. How relevant is the paper to answer the review \\ questions? \\ a. Very relevant \\ b. Somewhat relevant \\ c. Not relevant \\ Scoring system \\ $\mathrm{a}=1$ \\ $\mathrm{b}=0.5$ \\ $\mathrm{c}=0$ \\ $\mathrm{d}=0$ \\ Overall quality rating \\ High: 6-7 points \\ Medium: $3.5-5.5$ points \\ Low: $0-3$
}

ing or in a residential setting, but none of the respective samples were exclusively composed of older persons living in these kinds of settings, as such studies would not have been included for review. It is also worth mentioning that two samples were composed exclusively of older persons with a dementia diagnosis (Hellström 2009, McIntyre \& Reynolds 2012), two exclusively of older persons receiving palliative care (Gott et al. 2004, Sutton \& Coast 2012) and one exclu- sively of older persons with a mental disorder diagnosis (excluding dementia) (Martinsson et al. 2012). However, these conditions are not associated with any particular analytical theme or subtheme. It should be mentioned that only two studies included in their samples older persons from ethnic minorities, and only one included exclusively migrant carers (for a summary of these studies, please see Table S2 in the online version of this article).

\section{Findings}

Six analytical themes emerged from the synthesis of the findings of the reviewed papers: asking for care as a major challenge; ambivalence; (dis)engagement in decisions concerning care; multiple losses as outcomes of receiving social care; multiple strategies to cope with losses originated by the ageing process; properties of 'good care'.

\section{Asking for care as a major challenge}

Needing care is considered by older people to be a major shift in their lives (Twigg 2000, Valokivi 2005, Janlöv et al. 2006a). It is difficult for older persons to ask for care because care is associated with discontinuity in relation to the past, as well as with decline and later life (Valokivi 2005, Janlöv et al. 2006a). Asking for care from services, in particular, often creates tension in older persons given their unfamiliarity with the health and social care systems, which creates feelings of powerlessness (Valokivi 2005). However, asking for care from the informal network (relatives and friends) also can be problematic, as we will see in the next section.

\section{Ambivalence}

Some studies revealed that older people experience ambivalence with respect to asking for care/receiving care from relatives and friends (Lewinter 2003, Gott et al. 2004, Janlöv et al. 2006a,b, Tanner 2010b, Martinsson et al. 2012). This ambivalence results primarily from the conflict between the preference and recognised advantages of informal care and the worries of being a burden for the informal carers. From the point of view of older people, informal care is associated with comfort and security, but there is '(...) a limit to how much help one could expect from one's next of kin' (Janlöv et al. 2006a, p. 332). In other words, older people have to balance feelings of comfort and guilt, and one form of dealing with these feelings is to compensate the informal carers in different forms (Janlöv et al. 2006b). 
Ambivalence can also result from the conflict of choosing between informal care versus formal care (Gott et al. 2004, Janlöv et al. 2006a,b, ThemesslHuber et al. 2007). This ambivalence is related to the previous one, given that older persons may consider formal care when they start feeling that the informal care they are receiving is exceeding the carers' capacity or is producing harmful impacts on them. In this sense, older persons view formal care/public care as a form of relieving their informal carers. However, formal care, in contrast to informal care, may be perceived as threatening. For example, for many older persons, receiving home care represents a new and unknown situation, which is associated with having strangers in their homes (Janlöv et al. 2006a). Others feel reluctant to contact service providers because the older persons do not want to disturb the providers (who are perceived as being busy); there are also some older persons who feel humiliated, as they perceive receiving care as synonymous with dependence and frailty (ThemesslHuber et al. 2007).

\section{(Dis)engagement in decisions concerning care}

The issue of the older persons' (dis)engagement in decisions concerning care is addressed mostly in papers focused on relationships involving formal carers. It is important to differentiate the involvement in decisions regarding the choice of the care organisation/care package from the control over the actual delivery of care.

Regarding the (dis)engagement in decisions about the care package, some older persons constantly struggle to preserve their self-determination (by participating in decisions, staying in control of their care-receiving trajectories, etc.), while others are empowered by formal carers to make their own decisions. These carers try to fully understand the older persons' situations, needs, preferences and histories, involving them in the negotiations and decisions concerning the care package (Valokivi 2005, Breitholtz et al. 2012). Some older persons actively disengage from formal care services because they are dissatisfied with the system (Valokivi 2005). At the opposite end of the spectrum, some older persons do not have any influence in the decisions concerning the care package: they feel that they are not heard and that they are prevented from taking an active role in decision-making (Valokivi 2005, Breitholtz et al. 2012).

With respect to control over the actual delivery of care, some older persons who feel that their needs and preferences are overlooked. For example, some feel that home-care workers make decisions for them, providing care in a routine and rushed way and not allowing time for engagement (Themessl-Huber et al. 2007, Breitholtz et al. 2012). In some cases, the older persons make the initial contact with a service provider, but they do not assume a participatory role during the subsequent stages of the care relationship (Valokivi 2005).

The participation of older people in decisions concerning their care packages is an important issue, as they tend to associate choice with independence although independence can mean different things to different people (Rabiee 2013) - and participation with citizenship (Valokivi 2005). However, it is important to add that:

(...) being forced into choices because of lack of knowledge about available alternatives can compromise independence. (Rabiee 2013, p. 12)

\section{Multiple losses as outcomes of receiving social care}

Receiving care in later life is a process that normally goes hand in hand with the process of multiple losses over time as a consequence of physical and mental decline, such as loss of energy, loss of abilities and loss of social relations (Janlöv et al. 2006a, Nicholson et al. 2012a). However, there are some losses that result directly from the process of receiving social care; these are discussed next and should be viewed as negative aspects of the experience.

\section{Loss of independence}

In some cases, receiving social care may lead to a decrease in independence for older persons. For example, after a fall, an older person may have her/ his daily activities restricted to safe areas by her/his carers, which entails a loss of mobility and independence, and the older person may feel that the carer is being unnecessarily restrictive. The use of a walking aid, as recommended by the carer, may be viewed by the older persons as an external indicator of loss of independence (Stewart \& McVittie 2011).

\section{Loss of autonomy}

For some older persons, receiving social care means a loss of autonomy. Living with relatives from whom they receive social care allows for less 'room for manoeuvre' in making their own decisions (Barry 1995). Receiving care from relatives could lead the older person to passivity (e.g. not demanding more care) to avoid burdening them further (Lewinter 2003). For some, receiving care means being dependent on the knowledge of others for decision-making and reception of adequate care (Martinsson et al. 2012). 
Conversely, some older persons use their health conditions to 'manipulate' their carers (to obtain care from them), which can be seen as a way of exercising power (Forbat 2005). This evidence challenges the straightforward associations of 'carer' with 'independent' and 'older person receiving care' with 'dependent', '(...) as people struggle towards constructing differing levels of dependency with each other' (Forbat 2005, p. 126).

\section{Loss of confidence}

Starting to receive care produces a loss of confidence in the older persons' capacities and abilities (Stewart \& McVittie 2011, Martinsson et al. 2012) and is associated with feelings of vulnerability and powerlessness (Martinsson et al. 2012). As discussed earlier, receiving care is experienced by older people as a major shift in their lives, producing some insecurity and uncertainty about their present and future situations (Janlöv et al. 2006a).

\section{Loss of social identity}

The interactions of older people with health and social care workers often produce negative experiences (Tadd \& Calnan 2009, Stewart \& McVittie 2011). Some older persons complain:

(...) of being ignored, of being treated as objects, of having their need for privacy insufficiently recognised, of being humiliated and ridiculed and of inappropriate forms of address being used. (Tadd \& Calnan 2009, p. 140)

The loss of social identity can also be produced by a sense of loss of time and space for oneself, which derives from the 'caring gaze' (surveillance) under which some older persons live (Forbat 2005).

\section{Multiple strategies to cope with losses originated by the ageing process}

The following are strategies presented by the reviewed papers to deal with losses associated with the ageing process, in general, rather than to deal with losses originated directly by the process of receiving care.

\section{Maintaining a life}

Sustaining activities and relationships are two dimensions of maintaining a life. To be involved in daily activities and routines, some old and some completely new (although often adjusted), is important not only because such involvement keeps the body moving but also because it offers a sense of 'filling the day' (Nicholson et al. 2012a), that is, a sense of having a life (From et al. 2009, Hellström 2009, Tanner 2010a, McIntyre \&
Reynolds 2012, Nicholson et al. 2012a,b). Maintaining old relationships and/or creating new ones are also important in sustaining a connection with life (Tanner 2010b, Nicholson et al. 2012a).

These strategies can be grouped into a practical dimension of dealing with losses, but losses are also handled mentally/cognitively, as we will see (Janlöv et al. 2006a, Tanner 2010d). It is important to emphasise that social care can have an important role in this practical dimension of dealing with losses (Janlöv et al. 2006b, From et al. 2009, Tanner 2010a). For example, the support of carers may help older persons regain a sense of control over certain aspects of daily routine (Janlöv et al. 2006b).

\section{Preserving continuity with the past and sustaining a sense of self}

Many older persons struggle to nurture links to their past lives to maintain a sense of continuity of identity (Tanner 2010b, Stewart \& McVittie 2011) or a sense of 'being the same person', although with some adjustments. For example, keeping some 'life themes' alive (e.g. a life story marked by self-determination and independence) is a way of linking with past identities and, consequently, of preserving some continuity of identity (Tanner 2010b). Carers often support this connection with the past, particularly when these carers treat the older person as a person with a history (Janlöv et al. 2006b). The combination of practical strategies for maintaining a life and mental/cognitive strategies for preserving continuity with the past contributes to sustaining a sense of self (Janlöv et al. 2006a, Tanner 2010d). This demonstrates that many older persons, even in difficult situations, exercise agency through their actions aimed at sustaining a sense of self.

\section{Letting go}

To counterbalance the previous statement, it is important to note that, after a period of time in the 'keeping going' stage, some older persons make a transition to the 'letting go' stage, which is characterised by a passive attitude to the losses they experience (Nicholson et al. 2012b). In these cases, older people recognise that their lives are coming to an end (Nicholson et al. 2012b).

It is worth mentioning that only one paper addressed the responses of older people in relation to having their personal hygiene needs taken care of by others (Twigg 2000). This study revealed that most of the older persons accepted the circumstances, recognising that they have to live with it (many even welcomed the care they received). However, some still felt embarrassed with the situation. 
Boosting factors

This review allowed us to identify three main types of factors that 'boost' the strategies of dealing with multiple losses associated with the ageing process: individual, interactional and contextual factors.

Individual factors include physical and mental health (positive) life experiences, financial resources (Tanner 2010c) and cognitive skills, such as openness about one's vulnerabilities, the acceptance of care and support, putting life in perspective and not adopting the role of a victim (Janssen et al. 2011).

Interactional factors include supportive, empowering and reciprocal relationships with relatives, friends, professionals and even the society at large (e.g. tolerant attitudes towards older people) (Tanner 2010c, Janssen et al. 2011). With respect to professional practice, it is important to note that these factors are constrained by the organisational context in which it is undertaken (Tadd \& Calnan 2009).

Contextual or community boosting factors include the availability of services and other social benefits, such as cash for care (Tanner 2010c, Janssen et al. 2011), the availability of informal (unpaid) care (Sutton \& Coast 2012) and the social services' organisational culture (Walsh \& Shutes 2013).

It should be emphasised that the strategies to deal with losses associated with ageing can be boosted by social care, as shown earlier in relation to 'maintaining a life' and 'preserving continuity with the past and sustaining a sense of self'.

\section{Properties of 'good care'}

Properties of 'good care' are presented next; they derive from older persons' accounts of their own experiences receiving social care. These are properties anchored in real experiences and should therefore be viewed as positive aspects of the experience of receiving social care.

\section{Reciprocity}

One of the key properties of 'good care' is reciprocity. A care relationship is based on reciprocity when it is bidirectional/interdependent in which all elements of the relationship give and receive something (Forbat 2005, Dunér \& Nordström 2007, From et al. 2009, McIntyre \& Reynolds 2012, Walsh \& Shutes 2013). A reciprocal relationship is a balanced relationship in terms of power and contributes to the preservation of the autonomy of older persons (Forbat 2005). When reciprocity is absent or when the unbalance of power is too accentuated, the care relationship is conceived as 'bad' (From et al. 2009). This situation is avoidable, depending largely on the attitudes of carers:
The caregivers were illuminated as the key actors who determine whether the care could be experienced as good or bad. (From et al. 2009, p. 163)

\section{Respect and attentiveness}

Having respectful and attentive carers is also synonymous with 'good care'. Such carers respect the preferences and wishes of the older persons, treat them as capable persons and support and incentivise them to make their own decisions (Ware et al. 2003, From et al. 2009, McIntyre \& Reynolds 2012, Wilde \& Glendinning 2012, Walsh \& Shutes 2013).

Likewise, attentive carers are carers who pay attention to the needs of the older persons and are concerned with their situations (From et al. 2009, Sutton \& Coast 2012). The care relationships characterised by a sense of 'togetherness' are based on respectful and attentive interactions (McIntyre \& Reynolds 2012).

\section{Safety and security}

From the older persons' perspective, safe and secure care is that which is provided by carers with practical and social competencies (From et al. 2009), during sufficient time to allow mutual acquaintance (From et al. 2009), and with continuity, that is, provided by the same carer over a considerable period of time (Francis \& Netten 2004, From et al. 2009).

In general, safe and secure care requires trust within the care relationships through which the older persons feel that their carers are acting in their best interests (Francis \& Netten 2004, From et al. 2009, Hellström 2009). According to the Mental Capacity Act Code of Practice (2005) instituted in the United Kingdom, every adult is presumed to have the capacity to make his/her own decisions and should be supported in making them. In addition, individuals have the right to make apparently unwise decisions. The principle of 'best interests' should be applied only when a person is assessed as lacking capacity to make decisions.

\section{Discussion}

We defined three questions for this systematic review: How do older persons experience the reception of social care? What are the negative and positive aspects of these experiences? What are the factors that influence the experiences?

Concerning the first two questions, the findings of this review allow us to state that the reception of social care by older persons is positively experienced in some cases and negatively experienced in other cases. Only four studies offer explicit evidence that 
the reception of social care is experienced as both positive and negative by the same older persons (Forbat 2005, Valokivi 2005, Breitholtz et al. 2012, Martinsson et al. 2012).

The positive aspects of the experience of receiving social care include reciprocity, respect, attentiveness, safety and security (competent care, enough time for care, continuity of care, trust) as well as engagement in decisions concerning care. These aspects are valued by the older persons who participated in the reviewed studies. Conversely, the negative aspects of the experience of receiving social care include difficulties in asking for care (recognition of decline and non-familiarity with services), ambivalence (understanding the advantages of informal care, but not wanting to be a burden, receiving informal care versus formal care), disengagement from decisions concerning care and multiple losses resulting directly from the reception of social care (loss of independence, loss of autonomy, loss of confidence and loss of social identity).

Several observations can be made from these findings. All positive aspects and the last two negative aspects refer directly to the relational dimension of care (although some more than others) in which the attitudes and behaviour of the carers are crucial. Interestingly, the importance of the attitudes and behaviour of the carers was also emphasised in a systematic review on dignity in the care of older people (Gallagher et al. 2008). The first two negative aspects do not directly refer to the relationality of care, but rather to tensions and difficulties faced by older persons. This is the result of the embeddedness of the care relationships in cultures that put a high value on personal autonomy and independence (Twigg 2000, Barnes 2012), as well as in organisational systems characterised by formality and bureaucracy (Valokivi 2005). Some of these tensions and difficulties, such as those discussed in the Ambivalence section, are derived from a moral reasoning about 'what is the right thing to do'.

The positive aspects discussed above are consistent with the ethics of care perspective, as developed by Tronto, Sevenhuijsen and Engster. As mentioned in the Introduction, Tronto (1993) found that integrity of care' can be achieved only through the integration of four moral principles: attentiveness, responsibility, competence and responsiveness. In addition to these principles, Sevenhuijsen (1998) proposes trust and Engster (2007) proposes respect. Almost all of these principles can be found in the positive aspects of receiving care. On the other hand, those positive aspects may also be conceptualised as sources of dignity, especially 'dignity of identity' as defined by Nordenfelt in the Introduction. Lloyd et al. (2014) advocate that respectful and attentive care relationships are crucial to preserving dignity in later life.

Conversely, some of the negative aspects mentioned above, such as loss of independence, loss of autonomy, loss of confidence and loss of social identity, can be viewed as indicators of a lack of 'integrative care' or 'bad care'. Further, these negative aspects can also be perceived as threats to dignity, primarily to the 'dignity of identity'.

Thus, this systematic review suggests that the ethics of care and dignity are two relevant conceptual frameworks for understanding the experiences of receiving social care in later life, although they are subject to some criticism. For example, the ethics of care is criticised because of the presupposition that the care process is initiated by the carers, not fully explaining the role of the recipient of care in the decision (Fine 2007).

Regarding the third review question, the findings clearly show that the attitudes and behaviour of the carers play a crucial role in the way the elders experience the reception of social care (although other factors of individual, organisational and societal nature also play a non-negligible role). As mentioned earlier, the relational dimension of care is present in all the positive aspects and two negative aspects of the experience of receiving social care.

In this vein, we conclude that both positive and negative experiences of receiving social care relate, mostly, to the relational dimension of care. Receiving social care per se does not automatically imply a negative or a positive experience. Rather, it is the concrete form of social care provision, primarily the attitudes and behaviour of the carers, which determine whether the care is experienced as positive or negative. This is consistent with the assertion made by Tronto (2013, p. 166) that 'care is about relationships'.

This conclusion has implications for professional and non-professional practice and for social policy. If all older persons' carers consider in their daily practices the positive and negative aspects of the experience of receiving care as identified here, particularly those directly associated with the relational dimension of care, they are more likely to provide 'good care' or 'dignified care' and to prevent 'bad care' or 'undignified care'. However, we must not forget that 'good care practices' (professional and non-professional) can be effective and sustainable only if social and public policies ensure 'good conditions' - in terms of training/education, time for care, income/ cash for care, security and protection - in order for carers 'to do their job' with dignity. 
This review also confirms that the research on the older persons' experiences and perspectives of receiving social care, although important, is still limited, as it identifies some questions that have not received adequate attention and that could be addressed in future studies. For example, the reviewed papers do not offer a clear picture regarding the strategies which older persons use to deal with the losses they experience from receiving social care, although some studies suggest that the strategies used to deal with losses originated by ageing are also used to deal with losses originated directly by the process of receiving social care (Forbat 2005, Tanner 2010b, Stewart \& McVittie 2011, Nicholson et al. 2012b). On the other hand, it would be useful to conduct research on this topic throughout Europe rather than just the northern countries. In addition, studies comparing the cultural perspectives of different countries would be interesting, along with studies that include ethnic minorities and migrant care workers.

\section{Strengths and limitations}

There is a lack of consensus with respect to the methods for reviewing qualitative studies, mainly for synthesising their results. Nevertheless, our synthesis, based on 'thematic synthesis' (Thomas \& Harden 2008), points to their potential value, as we were able to compare the empirical data within and across the studies and, as a result, generated analytical themes and a conceptual development beyond what was achieved in each individual study. Thematic synthesis is similar to meta-ethnography and 'meta-synthesis'. However, as suggested by Thomas and Harden (2008), when specific review questions are defined, as happened in this review, they are used as a 'theoretical framework' which interrogates a descriptive synthesis and generates analytical themes, instead of 'third-order interpretations' typical of meta-ethnographies. The primary difficulty we faced throughout the synthesis process had to do with the creation of the analytical themes due to heterogeneity of the samples (typical in qualitative studies).

\section{Acknowledgements}

This paper has been partially supported by the Portuguese Foundation for Science and Technology (FCT).

\section{References}

Barnes M. (2012) Care in Everyday Life. An Ethic of Care in Practice. The Policy Press, Bristol, UK.
Barry J. (1995) Care-need and care-receivers. Views from the margins. Women's Studies International Forum 18 (3), 361-374.

Breitholtz A., Snellman I. \& Fagerberg I. (2012) Older people's dependence on caregivers' help in their own homes and their lived experiences of their opportunity to make independent decisions. International Journal of Older People Nursing 8 (2), 139-148.

Britten N., Campbell R., Pope C., Donovan J., Morgan M. \& Pill R. (2002) Using meta-ethnography to synthesize qualitative research: a worked example. Journal of Health Services Research and Policy 7 (4), 209-215.

Dunér A. \& Nordström M. (2007) The roles and functions of the informal support networks of older people who receive formal support: a Swedish qualitative study. Ageing and Society 27, 67-85.

Edmonson R. \& Kondratowitz H. (2009) Establishing a humanistic gerontology - challenges and Opportunities. In: R. Edmondson \& H. von Kondratowitz (Eds) Valuing Older People. A Humanist Approach to Ageing, pp. 1-20. The Policy Press, Bristol, UK.

Engster D. (2007) The Heart of Justice: Care Ethics and Political Theory. Oxford University Press, Oxford, UK.

Fine M. (2007) A Caring Society? Care and the Dilemmas of Human Service in the 21st Century. Palgrave Macmillan, Sydney.

Forbat L. (2005) Embedding difficulties in talk about care relationships. In: L. Forbat (Ed.) Talking About Care, pp. 107-128. The Policy Press, Bristol, UK.

Francis J. \& Netten A. (2004) Raising the quality of home care: a study of service users' views. Social Policy and Administration 38 (3), 290-305.

From I., Johansson I. \& Athlin E. (2009) The meaning of good and bad care in the community care: older people's lived experiences. International Journal of Older People Nursing 4, 156-165.

Gallagher A., Li S., Wainwright P., Jones I. \& Lee D. (2008) Dignity in the care of older people - a review of the theoretical and empirical literature. BMS Nursing 7, 11.

Gorman M. (2000) Development and the rights of older people. In: J. Randel, T. German \& D. Ewing (Eds) The Ageing and Development Report: Poverty, Independence and the World's Older People, pp. 3-21. Earthscan Publications Ltd., London, UK.

Gott M., Seymour J., Bellamy G., Clark D. \& Ahmedzai S. (2004) Older people's views about home as a place of care at the end of life. Palliative Medicine 18, 460-467.

Hellström I. (2009) Dignity and older spouses with dementia. In: L. Nordenfelt (Ed.) Dignity in Care for Older People, pp. 99-118. Wiley-Blackwell, Oxford, UK.

Janlöv A., Hallberg I. \& Petersson K. (2006a) The experience of older people of entering into the phase of asking for public home help - a qualitative study. International Journal of Social Welfare 14, 326-336.

Janlöv A., Hallberg I. \& Petersson K. (2006b) Older persons' experience of being assessed for and receiving public home help: do they have any influence over it? Health and Social Care in the Community 14 (1), 26-36.

Janssen B., Regenmortel T. \& Abma B. (2011) Identifying sources of strength: resilience from the perspective of older people receiving long-term community care. European Journal of Ageing 8, 145-156.

Lewinter M. (2003) Reciprocities in caregiving relationships in Danish elder care. Journal of Aging Studies 17, 357-377. 
Lloyd L. (2012) Health and Care in Ageing Societies. A New International Approach. The Policy Press, Bristol, UK.

Lloyd L., Calnan M., Cameron A., Seymour J. \& Smith R. (2014) Identity in the fourth age: perseverance, adaptation and maintaining dignity. Ageing and Society 34, 1-19.

Martinsson G., Fagerberg I., Lindholm C. \& Wiklund-Gustin L. (2012) Struggling for existence life situation experiences of older persons with mental disorders. International Journal of Qualitative Study of Health and Well-Being 7, 18422.

McIntyre A. \& Reynolds F. (2012) There's no apprenticeship for Alzheimer's: the caring relationship when an older person experiencing dementia falls. Ageing and Society 32, 873-896.

Nicholson C., Meyer J., Flatley M. \& Holman C. (2012a) The experience of living at home with frailty in old age: a psychosocial qualitative study. International Journal of Nursing Studies 50 (9), 1172-1179.

Nicholson C., Meyer J., Flatley M., Holman C. \& Lowton K. (2012b) Living on the margin: understanding the experience of living and dying with frailty in old age. Social Science and Medicine 75, 1426-1432.

Nolan M., Davies S., Brown J., Keady J. \& Nolan J. (2004) Beyond "person-centred" care: a new vision for gerontological nursing. International Journal of Older People Nursing, in association with Journal of Clinical Nursing 13 (3a), 45-53.

Nordenfelt L. (2009) The concept of dignity. In: L. Nordenfelt (Ed.) Dignity in Care for Older People, pp. 26-53. Wiley-Blackwell, Oxford, UK.

Petticrew M. \& Roberts H. (2006) Systematic Reviews in the Social Sciences. A Practical Guide. Blackwell Publishing, Malden.

Rabiee P. (2013) Exploring the relationships between choice and independence: experiences of disabled and older people. British Journal of Social Work 43, 1-17.

SCIE (2010) SCIE Systematic Research Reviews: Guidelines, $2^{\text {nd }}$ edn. Social Care Institute for Excellence, London.

Sevenhuijsen S. (1998) Citizenship and the Ethics of Care: Feminist Considerations of Justice, Morality and Politics. Routledge, London, UK.

Sipilä J. \& Kröger T. (2005) Editorial introduction: European families stretched between the demands of work and care. In: T. Kröger \& J. Sipilä (Eds) Overstretched. European Families Up Against the Demands of Work and Care, pp. 1-8. Blackwell Publishing, Oxford, UK.

Stewart J. \& McVittie C. (2011) Living with falls: housebound older people's experiences of health and community care. European Journal of Ageing 8, 271-279.

Sutton E. \& Coast J. (2012) 'Choice is a small word with a huge meaning': autonomy and decision making at the end of life. Policy and Politics 40 (2), 211-226.

Tadd W. \& Calnan M. (2009) Caring for older people: why dignity matters - the European experience. In: L. Norden- felt (Ed.) Dignity in Care for Older People, pp. 119-145. Wiley-Blackwell, Oxford, UK.

Tanner D. (2010a) Keeping going. In: D. Tanner (Ed.) Managing the Ageing Experience. Learning from Older People, pp. 55-82. The Policy Press, Bristol, UK.

Tanner D. (2010b) Staying me. In: D. Tanner (Ed.) Managing the Ageing Experience. Learning from Older People, pp. 83116. The Policy Press, Bristol, UK.

Tanner D. (2010c) The slippery slope. In: D. Tanner (Ed.) Managing the Ageing Experience. Learning from Older People, pp. 117-154. The Policy Press, Bristol, UK.

Tanner D. (2010d) Sustaining the self. In: D. Tanner (Ed.) Managing the Ageing Experience. Learning from Older People, pp. 155-186. The Policy Press, Bristol, UK.

Themessl-Huber M., Hubbard G. \& Munro P. (2007) Frail older people's experiences and use of health and social care services. Journal of Nursing Management 15, 222-229.

Thomas J. \& Harden A. (2008) Methods for the thematic synthesis of qualitative research in systematic reviews. BMC Medical Research Methodology 8, 45.

Tronto J.C. (1993) Moral Boundaries: A Political Argument for an Ethic of Care. Routledge, London, UK.

Tronto J.C. (2013) Caring Democracy. Markets, Equality, and Justice. New York University Press, New York and London.

Twigg J. (2000) Bathing, washing and the management of personal care. In: J. Twigg (Ed.) Bathing, the Body and Community Care, pp. 45-76. Routledge, London, UK.

Valokivi H. (2005) Participation and citizenship of elderly persons. Social Work in Health Care 39 (1-2), 181-207.

Walsh K. \& Shutes I. (2013) Care relationships, quality of care and migrant workers caring for older people. Ageing and Society 33, 393-420.

Ware T., Matosevic T., Hardy B., Knapp M., Kendall J. \& Forder J. (2003) Commissioning care services for older people in England: the view from care managers, users and carers. Ageing and Society 23, 411-428.

Wilde A. \& Glendinning C. (2012) 'If they're helping me then how can I be independent?' The perceptions and experience of users of home-care re-ablement services. Health and Social Care in the Community 20 (6), 583-590.

\section{Supporting Information}

Additional supporting information may be found in the online version of this article at the publisher's web-site. 